\title{
Article \\ CRISPR Deletion of a SVA Retrotransposon Demonstrates Function as a cis-Regulatory Element at the TRPV1/TRPV3 Intergenic Region
}

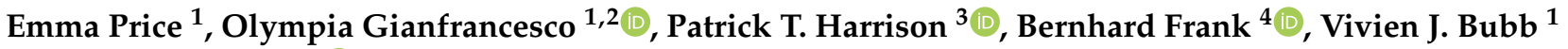 \\ and John P. Quinn ${ }^{1, *}$ (D) \\ 1 Department of Pharmacology and Therapeutics, Institute of Systems, Molecular \& Integrative Biology, \\ University of Liverpool, Liverpool L69 3GE, UK; emma.price2@nih.gov (E.P.); \\ olympia.gianfrancesco@igmm.ed.ac.uk (O.G.); jillbubb@liverpool.ac.uk (V.J.B.) \\ 2 MRC Human Genetics Unit, Institute of Genetics and Molecular Medicine, University of Edinburgh, \\ Edinburgh EH4 2XU, UK \\ 3 Department of Physiology, BioSciences Institute, University College Cork, Cork, Ireland; p.harrison@ucc.ie \\ 4 Department of Pain Medicine, Walton Centre NHS Foundation Trust, Liverpool L9 7LJ, UK; \\ bernhard.Frank@liverpool.ac.uk \\ * Correspondence: jquinn@liverpool.ac.uk; Tel.: +44-0151-794-5498
}

Citation: Price, E.; Gianfrancesco, O.; Harrison, P.T.; Frank, B.; Bubb, V.J.; Quinn, J.P. CRISPR Deletion of a SVA Retrotransposon Demonstrates Function as a cis-Regulatory Element at the TRPV1/TRPV3 Intergenic Region. Int. J. Mol. Sci. 2021, 22, 1911. https://doi.org/10.3390/ijms 22041911

Academic Editor: Michael Hausmann Received: 15 January 2021

Accepted: 5 February 2021

Published: 15 February 2021

Publisher's Note: MDPI stays neutral with regard to jurisdictional claims in published maps and institutional affiliations.

Copyright: (C) 2021 by the authors Licensee MDPI, Basel, Switzerland. This article is an open access article distributed under the terms and conditions of the Creative Commons Attribution (CC BY) license (https:// creativecommons.org/licenses/by/ $4.0 /)$.

\begin{abstract}
SINE-VNTR-Alu (SVA) retrotransposons are a subclass of transposable elements (TEs) that exist only in primate genomes. TE insertions can be co-opted as cis-regulatory elements (CREs); however, the regulatory potential of SVAs has predominantly been demonstrated using bioinformatic approaches and reporter gene assays. The objective of this study was to demonstrate SVA cisregulatory activity by CRISPR (clustered regularly interspaced short palindromic repeats) deletion and subsequent measurement of direct effects on local gene expression. We identified a region on chromosome 17 that was enriched with human-specific SVAs. Comparative gene expression analysis at this region revealed co-expression of TRPV1 and TRPV3 in multiple human tissues, which was not observed in mouse, highlighting key regulatory differences between the two species. Furthermore, the intergenic region between TRPV1 and TRPV3 coding sequences contained a human specific SVA insertion located upstream of the TRPV3 promoter and downstream of the $3^{\prime}$ end of TRPV1, highlighting this SVA as a candidate to study its potential cis-regulatory activity on both genes. Firstly, we generated SVA reporter gene constructs and demonstrated their transcriptional regulatory activity in HEK293 cells. We then devised a dual-targeting CRISPR strategy to facilitate the deletion of this entire SVA sequence and generated edited HEK293 clonal cell lines containing homozygous and heterozygous SVA deletions. In edited homozygous $\triangle$ SVA clones, we observed a significant decrease in both TRPV1 and TRPV3 mRNA expression, compared to unedited HEK293. In addition, we also observed an increase in the variability of mRNA expression levels in heterozygous $\triangle$ SVA clones. Overall, in edited HEK293 with SVA deletions, we observed a disruption to the co-expression of TRPV1 and TRPV3. Here we provide an example of a human specific SVA with cis-regulatory activity in situ, supporting the role of SVA retrotransposons as contributors to species-specific gene expression.
\end{abstract}

Keywords: retrotransposon; SVA; cis-regulatory element; TRPV1; TRPV3; CRISPR; gene expression

\section{Introduction}

SINE-VNTR-Alus (SVAs) are the evolutionarily youngest family of transposable elements (TEs) currently characterized within the human genome. The SVA family emerged throughout primate evolution (Figure 1A) and belong to a group of TEs termed non-long terminal repeat (LTR) retrotransposons (which also includes long interspersed nuclear element 1 (LINE-1) and $A l u$ ), which collectively remain the only actively mobile TEs in the human genome [1,2]. Mobilization of non-LTR TEs leads to novel insertions which 
contribute to genome sequence and structure-ultimately contributing to genome evolution [3-5]. The insertion site of TEs can affect gene regulation through multiple mechanisms including the introduction of transcription factor binding sites (TFBSs), novel transcriptional start sites (TSS), alternative splicing, exonization, and alterations to epigenetic marks including DNA methylation and histone modifications [6-10]. Thus, novel TE insertions can be co-opted for new regulatory functions which drives species-specific gene expression. In recent years, attention has turned towards the regulatory impact of SVAs in the human genome. Previously, we have demonstrated the transcriptional regulatory properties of isolated SVA sequences utilizing reporter gene assays [11,12]. More recently, bioinformatic studies utilizing ChIP-seq and RNA-seq datasets generated from liver across different primate species have identified that newly evolved (i.e., species-specific) cis-regulatory elements (CREs) were enriched in SVA sequences, highlighting SVAs as potentially important contributors to gene regulation in primates $[13,14]$. Candidate SVA CREs were verified using reporter gene assays, which lent support to the hypothesis that SVAs can have regulatory properties but this did not necessarily confirm regulatory function in situ in the human genome, and more specifically in the context of endogenous genes $[13,14]$.

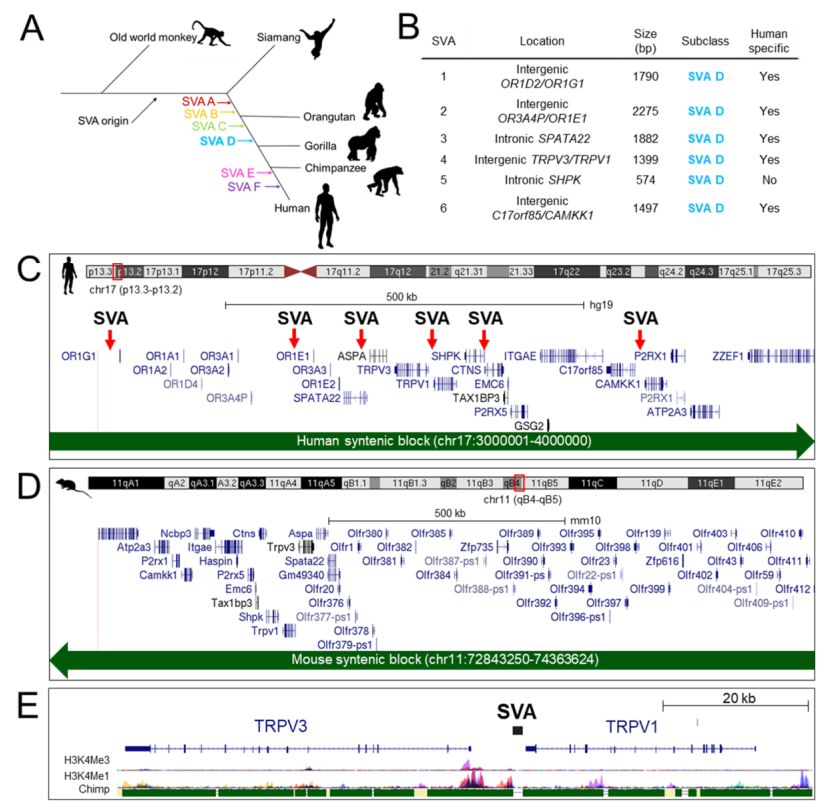

Figure 1. Chr17p13.2 is enriched for human specific SVA D insertions. (A) Schematic showing the emergence of short interspersed nuclear element-variable number tandem repeat (SINE-VNTR)-Alu (SVA) subclasses throughout primate evolution. SVA subclasses A, B, and C are evolutionarily older and are found in multiple primate species, whereas subclasses SVA E and SVA F emerged following divergence with the chimpanzee last common ancestor and are therefore human specific (adapted from Wang et al. 2005) (B) Summary of SVA D insertions at chr17p13.2. (C) A gene dense region (chr17:3000001-4000000, hg19) at chr17p13.2 contains 6 SVA D insertions. (D) The syntenic region in the mouse genome (chr11:72843250-74363624, mm10) contains orthologous genes conserved at chr11qB4-B5 in the opposite orientation to that displayed in the human genome. (E) UCSC image showing human specific SVA insertion at the TRPV1/TRPV3 intergenic region respect to adjacent genes. H3K4Me3 and H3K4Me1 histone marks are shown, highlighting regulatory domains. Conservation with the chimpanzee genome is also provided to assess SVA status as primate or human specific.

A direct approach to address the role of candidate regulatory domains in situ can be performed through CRISPR (clustered regularly interspaced short palindromic repeats)/Cas9 (CRISPR associated protein 9)-mediated deletion of entire TE sequences. An exemplar of this approach was seen in the investigation of the etiology of neurodegenerative disorder X-linked dystonia parkinsonism (XDP). In this study, an XDP-specific 
SVA insertion was shown to cause intron retention and reduced expression of the gene TAF1, which was causative of the disorder $[15,16]$. Upon CRISPR/Cas9 deletion of the SVA in patient derived induced pluripotent stem cells (iPSCs), aberrant splicing and TAF1 expression were rescued, implicating the SVA in disease-associated gene regulation. Herein, our specific aim was to extend this approach and demonstrate cis-regulatory effects of an SVA in a non-disease context, by highlighting the contribution of an endogenous SVA to human specific gene expression.

We and others have previously demonstrated that SVAs preferentially insert into gene dense regions and several regions across the human genome are enriched for SVA insertions [17]. One such region previously identified was a $1 \mathrm{Mb}$ region (chr17:3000001-4000000, hg19) on chromosome 17 (chr17p13.2), which contained six SVA insertions (Figure 1B). These insertions were SVA subclass D, which is a subclass that shares some insertions with gorilla and chimpanzee but the majority $(67.5 \%)$ are human specific [3]. Furthermore, SVA D comprises $44.4 \%$ of all SVAs in the human genome [17], highlighting this subclass as particularly active throughout human evolution. It has been established that there is a strong bias of human specific SVAs inserting into regions already containing SVAs [11,12,17]. All SVAs at chr17p13.2 were subclass SVA D (Figure 1C), with 5 out of 6 being full length and human-specific (Figure 1B), highlighting chr17p13.2 as an area of the genome that has been tolerant to multiple SVA insertions throughout recent human evolution and an area of the genome where human-specific gene regulation via SVA-mediated activity may have evolved. Given the enrichment of human-specific SVAs at this region, in such close proximity to many genes, it provided a region of the genome with multiple potential human specific CREs to explore further.

To identify a candidate SVA at this location with the potential to function as a cisregulatory element, we explored the location of SVAs at chr17p13.2 with respect to their adjacent genes and at the same time, compared RNA-seq data from humans and mice. This approach identified an SVA insertion, proximal to genes TRPV1 and TRPV3, which displayed human-specific expression patterns. TRPV1 and TRPV3 encode polymodal transient receptor potential channels which enable thermosensory perception and have well established roles in pain and inflammation [18,19]. Our preliminary data obtained from reporter gene assays suggested this SVA was indeed regulatory, and thus highlighted this SVA as a candidate for further study. To determine cis-regulatory activity in situ, we devised a dual-targeting CRISPR/Cas9 strategy to delete the SVA in the HEK293 cell line and measured effects on TRPV1 and TRPV3 gene expression. In this study, we deleted the entire SVA sequence in multiple HEK293 clones and demonstrated gene expression changes in comparison to unedited cells, providing in situ functional data that supported the role of SVAs as CREs in the human genome and highlighted their role in the evolution of gene regulation in humans.

\section{Results}

2.1. Intergenic Region between TRPV1 and TRPV3 Contains a Human Specific SVA Insertion Predicted to Function as a Regulatory Domain

In the preliminary bioinformatic analysis of SVA insertions across the chr17p13.2 region (Figure 1C), the SVA at the TRPV1 and TRPV3 locus was notable as it was located within the intergenic region containing the TRPV3 promoter and directly adjacent to other prominent histone marks indicative of regulatory domains (Figure 1E). A more detailed overview of other SVA insertions in respect to other genes is provided in Figure S1. Analysis showed that the SVA and both TRPV1 and TRPV3 coding sequences, were all encoded on the antisense strand of DNA, with the SVA located approximately 400 bp downstream of the TRPV1 $3^{\prime} \mathrm{UTR}$ and $5.7 \mathrm{~kb}$ upstream of the $5^{\prime}$ TRPV3 TSS (Figure 2A). The intergenic region contained numerous repetitive DNA sequences that were conserved with other primate species (Figure $2 \mathrm{~A}, \mathrm{~B}$ ). In total, $73 \%$ of the $7.4 \mathrm{~kb}$ intergenic sequence was comprised of TEs including multiple SINEs (specifically Alus) (36\%) and LINEs (12\%) (Figure 2B). The single SVA insertion (chr17:3466973-3468374, hg19) was the largest TE (1402 bp) in this region, accounting for a substantial proportion (19\%) of the intergenic sequence (Figure $2 \mathrm{~B}$ ). 
In addition, the SVA was directly adjacent to an evolutionary conserved region (ECR) (chr17:3466258-3466820 hg19) containing a mammalian-wide interspersed repeat (MIR) (Figure 2A). MIRs are the most ancient family of TEs in the human genome, enriched for TFBSs and have been shown to function as enhancers in vitro [20]. ENCODE histone data overlaid across this ECR was indicative of regulatory activity and it was also listed as a candidate CRE of TRPV1 and TRPV3 within ENCODE (ENCODE acc. no. EH38E1841572) (Figure 2A). No histone data were available for the SVA itself because larger repetitive sequences are difficult to map in short read sequence data, thus they are often excluded from such analyses (Figure 2A). Nevertheless, the available bioinformatic data supported that the ECR was a functional CRE and we reasoned that its modulation in humans may be impacted upon by the adjacent human specific SVA insertion.

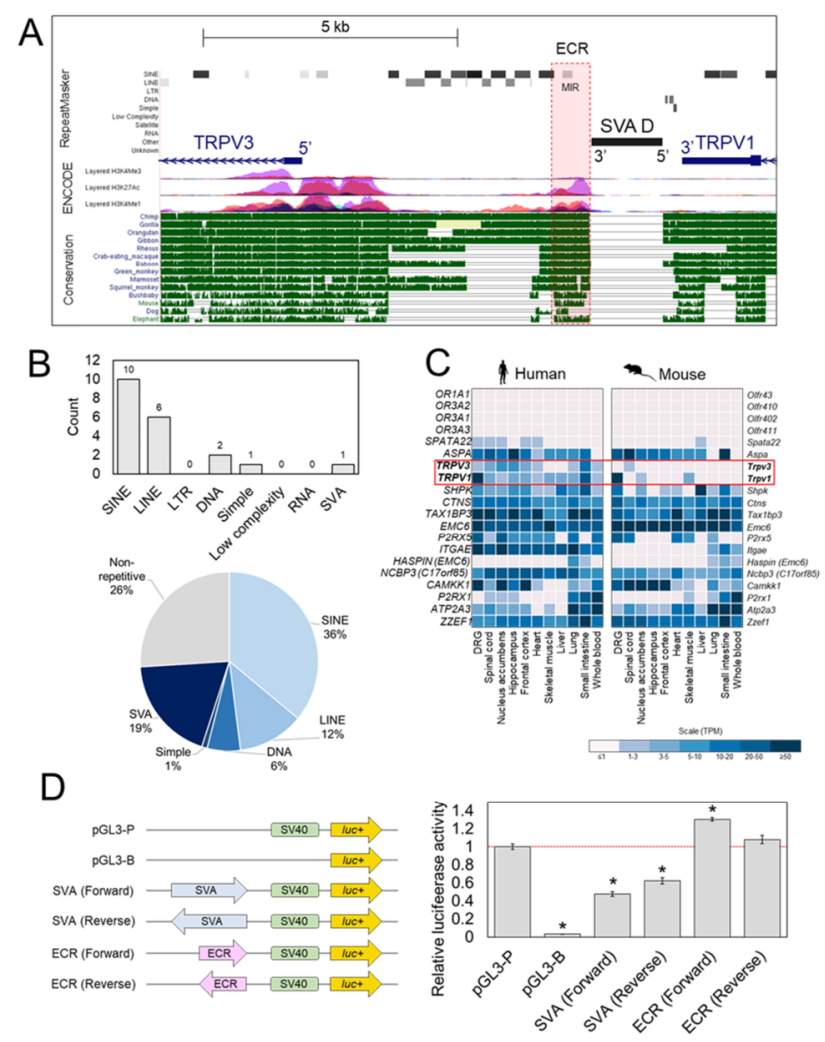

Figure 2. Intergenic region between TRPV1 and TRPV3 contains human-specific SVA insertion predicted to function as regulatory domain. (A) UCSC image of intergenic region between TRPV1 and TRPV3. RepeatMasker displays repetitive DNA including SVA insertion (ECR containing MIR element is highlighted in red). (B) Analysis of TE composition of the intergenic sequence between TRPV1 and TRPV3. (C) RNA-seq data showing normalized gene expression values (expressed as transcripts per million; TPM) of genes encoded at chr17p13.2 in human and orthologous genes encoded at chr11qB4-B5 in mouse. (D) Relative luciferase activity of pGL3-P reporter gene constructs with SVA (chr17:3466973-3468374) and ECR sequences (chr17:3466258-3466820) cloned upstream of the minimal SV40 promoter (green box) and transfected into HEK293 $(n=4)$. ${ }^{*} p$-value $<0.05$ (2-sample $t$-test).

To address human-specific gene expression, we compared the expression of all protein coding genes encoded at chr17p13.2 (Figure 1C) with orthologous genes encoded at the syntenic region (33.4\% of bases and $99.4 \%$ of span) chr11(qB4-qB5) in the mouse genome (Figure 1D). Utilizing data from an RNA-seq study which compared gene expression across multiple tissues between human and mouse [21], expression differences in various genes at chr17p13.2 were noted (e.g., ITGAE, P2RX1, P2RX1) (Figure 2C). However, the regulation of TRPV1 and TRPV3 was our focus, due to the organization of the surrounding regulatory domains with respect to the SVA insertion (Figure 2A). We observed widespread 
expression of TRPV1 and TRPV3 across multiple human tissues in comparison to a tissue restricted expression profile in the mouse (Figure 2C). We observed $h T R P V 1$ expression was generally at relatively low levels but with high expression in dorsal root ganglia (DRG). Similarly, mTrpo1 was also expressed at relatively high levels in DRG, however mTRPV1 expression was in general more restricted, with low levels of expression only documented in nucleus accumbens and skeletal muscle. $h T R P V 3$ expression was seen in most tissues analyzed however $m T r p v 3$ was restricted to spinal cord. This analysis also highlighted the co-expression of TRPV1 and TRPV 3 in many human tissues compared to mice, suggesting differences in key regulatory mechanisms at the molecular level. We hypothesized that the SVA may be functional as a cis-regulatory domain contributing to the regulatory differences of TRPV1 and TRPV3 observed in humans.

\subsection{Reporter Gene Assays Support Regulatory Potential of SVA at TRPV1/TRPV3 Locus}

To confirm the regulatory potential of the SVA and the adjacent ECR, both sequences were cloned into the reporter gene construct pGL3-P, upstream of the minimal SV40 promoter in both forward and reverse orientations - with forward resembling the endogenous orientation of the SVA with respect to TRPV1 and TRPV3 (Figure 2D). The sequences and coordinates of the cloned SVA and ECR fragments are given in Supplementary File 2. Luciferase activity was measured $48 \mathrm{~h}$ post-transfection. In HEK293 transfected with the SVA reporter gene constructs, a statistically significant 2 -fold decrease $(p<0.05)$ in luciferase activity was observed when the SVA was cloned in the forward orientation. Similarly, a smaller yet still significant 1.6-fold decrease $(p<0.05)$ when cloned in the reverse orientation when compared to the unmodified pGL3-P vector was observed, indicating the SVA was functional as a transcriptional regulatory domain in this cell line, independent of orientation (Figure 2D). The ECR reporter gene construct displayed a small 1.3-fold increase $(p<0.05)$ in luciferase activity when the ECR was cloned in the forward orientation but no difference was observed when the ECR was cloned in the reverse orientation when compared to unmodified pGL3-P (Figure 2D).

\subsection{Dual-Targeted CRISPR/Cas9 Deletion of the SVA in HEK293}

To address the hypothesis in the context of the endogenous genes, expression of TRPV1 and TRPV3 was confirmed in the HEK293 cell line (Figure S2). HEK293 was chosen as a model cell line to conduct CRISPR due to its high transfection efficiency, which was found to be a limiting factor in the genome editing efficiency in other cell lines we tested (e.g., HAP1 and SH-SY5Y) during early stages of protocol development (data not shown). Upon confirmation both genes were active in this cell line, a dual-targeted CRISPR strategy to delete the entire SVA sequence in HEK293 cells was developed to subsequently measure the potential impact on TRPV1 and TRPV3 expression (Figure 3A) [22]. Two guide RNAs (gRNAs) were designed, which targeted sequences $66 \mathrm{bp}$ downstream and $213 \mathrm{bp}$ upstream of the SVA, and cloned into the Cas9 expression vector pSpCas9(BB)-2A-GFP, resulting in two separate vectors each containing a single gRNA sequence (Figure 3A) [23]. The dual-target strategy, based on co-transfection of the two independent Cas9 vectors each containing a specific gRNA tag, was predicted to result in generation of two double strand breaks (DSBs) at positions chr17:3563606 and chr17:3565282 (hg19), following which the intermediate sequence containing the SVA would be lost and the ends repaired via nonhomologous end joining (NHEJ). This approach was designed to facilitate the deletion of a $1677 \mathrm{bp}$ sequence containing the SVA (1402 bp) (Figure 3A).

Edited HEK293 cells were expanded as clonal cells lines and CRISPR-edited genetic regions were screened via PCR to identify clones containing the desired SVA deletions (Figure 3B). PCR products for unedited (containing SVA) and edited (deleted SVA) regions were $2486 \mathrm{bp}$ and $808 \mathrm{bp}$ in length, respectively (Figure 3B). Following transfection, 215 clonal cell lines were screened in total. Three independent clonal cell lines $(<2 \%)$ amplified only edited PCR products, indicating all SVA alleles were successfully deleted, and were termed homozygous $\triangle$ SVA clones. In addition to the desired homozygous $\triangle$ SVA HEK293 
genotype, several clonal cell lines (10\%) amplified both unedited and edited PCR products, indicating that these clones carried an intact SVA and an SVA deletion (Figure 3B). These clones were termed heterozygous $\triangle$ SVA clones and three (chosen at random) were included in subsequent analysis. As an additional control, HEK293 clones transfected with nontarget gRNAs (ntgRNAs) were also generated; these guides were specifically designed to not recognize any human DNA sequence, thus should not guide the Cas9 to any specific sequence or result in any modifications.

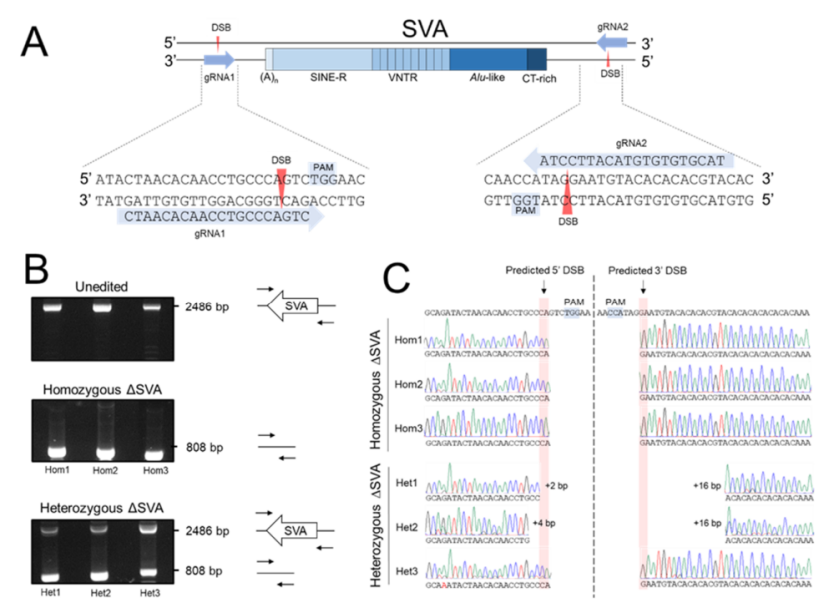

Figure 3. Generation of clonal cell lines containing knockout of entire SVA sequence. (A) Schematic of dual-gRNA strategy designed to delete entire SVA sequence. gRNAs were designed to anneal to short 20 bp sequences upstream of protospacer adjacent motif (PAM) sites ( $5^{\prime}$-NGG-3 $\left.{ }^{\prime}\right)$ situated on either side of the SVA. Cas9 generates double strand breaks 3-4 bp upstream of the PAM sequence. The SVA is then excised before the two ends are repaired typically by NHEJ, resulting in deletion of the SVA. Schematic not to scale. (B) PCR screening showing isolation of several homozygous $\triangle$ SVA clones with all SVAs deleted and heterozygous $\triangle$ SVA clones demonstrating presence and absence of SVA alleles. (C) Sequencing analysis across breakpoints in PCR products.

Sequencing of the PCR products across the predicted DSB breakpoints was performed in all homozygous and heterozygous $\Delta$ SVA clones. In all three homozygous $\Delta$ SVA clones, the breakpoints were identified at the predicted DSB sites with no indels, highlighting effective and accurate repair at the edited sites (Figure 3C). This data confirmed the predicted modifications had been generated in these clones, with a precise deletion of $1677 \mathrm{bp}$ containing the SVA. Sequencing of the heterozygous $\triangle$ SVA clones demonstrated that only clone 3 had breakpoints at the predicted DSB sites. Clone 1 and 2 carried slightly larger deletions (Figure $3 \mathrm{C}$ ). Breakpoints in clone 2 extended $2 \mathrm{bp}$ and $16 \mathrm{bp}$ beyond the predicted gRNA1 and gRNA2 DSB sites, respectively. Clone 1 carried the largest deletion, with breakpoints extending $4 \mathrm{bp}$ and $16 \mathrm{bp}$ beyond the predicted gRNA1 and gRNA2 DSB sites, respectively. These data confirm that the desired modifications were successfully generated in homozygous $\triangle$ SVA clones and additional modifications had occurred in heterozygous $\triangle$ SVA clones which were also taken forward for gene expression analysis.

\subsection{TRPV1 and TRPV3 Expression Was Disrupted in CRISPR Edited HEK293 Clones Containing SVA Deletions}

To assess if the SVA was functional as a CRE, total mRNA expression levels of adjacent genes TRPV1 and TRPV3 were measured using qPCR (Figure 4). Validation of qPCR products and primer efficiencies are shown in Figure S2. Expression levels in edited clonal cell lines were compared against unedited HEK293. All relative expression levels were normalized against reference gene $A C T B$. Relative expression values were plotted as log2 fold change to enable comparative visualization of increases and decreases in expression levels. HEK293 cells transfected with ntgRNAs showed negligible difference in TRPV3 levels $(p>0.05)$, when compared to unedited cells, indicating that any changes observed in 
edited cells could be directly attributed to deletion of the SVA and not due to transfection of CRISPR machinery without genome modification (Figure 4A). We did observe a slight decrease in TRPV1 in ntgRNA cells, however this was negligible and not determined to be statistically significant $(p>0.05)$ (Figure $4 \mathrm{~A})$. In homozygous $\triangle$ SVA clones, a significant decrease in TRPV3 mRNA expression $(p<0.05)$ was observed (Figure $4 \mathrm{~B})$. A decrease in TRPV1 expression was also seen that was determined to be statistically significant $(p<0.05)$, however it was relatively small compared to unedited cells and also similar to the small decrease in cells transfected with ntgRNAs. This implied that, under the experimental conditions employed, TRPV1 expression between individual HEK293 clones was minimally affected by the presence or absence of the SVA. However, a consistent and much greater decrease in TRPV3 expression was observed. These results provided evidence which supported the role of this SVA as a CRE at the intergenic region between TRPV1 and TRPV3 in HEK293.

A
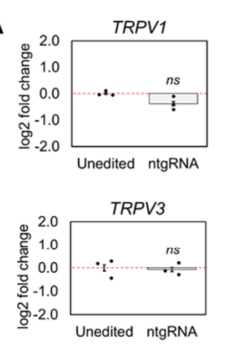

C

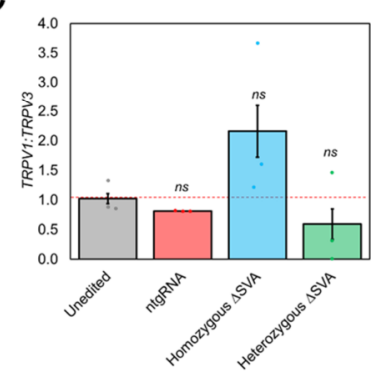

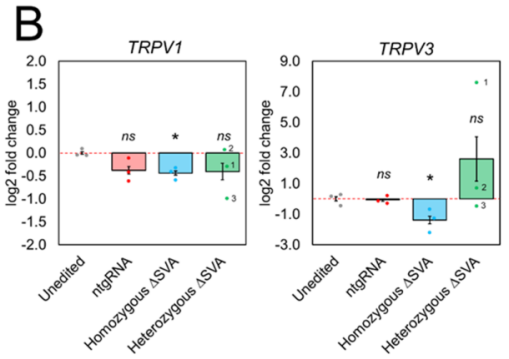

$\mathrm{D}$

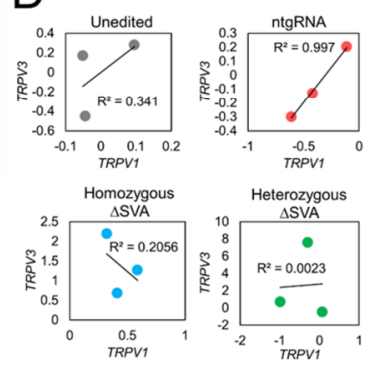

Figure 4. Clustered regularly interspaced short palindromic repeats (CRISPR)-mediated deletion of SVA in HEK293 disrupts co-expression of TRPV1 and TRPV3 mRNA. (A) TRPV1 and TRPV3 mRNA expression in cells transfected with ntgRNAs compared to unedited HEK293. (B) mRNA expression in unedited cells, ntgRNA controls, homozygous $\triangle$ SVA clones, and heterozygous $\triangle$ SVA clones. (C) Ratio of TRPV1:TRPV3 mRNA levels. (D) Pearson's correlation of TRPV1 and TRPV3 mRNA in all cell lines. Abbreviations; not significant (ns), ${ }^{*}$-value $<0.05$ (2-sample $t$-test).

In contrast with homozygous $\triangle$ SVA HEK293 clones, which had shown relatively consistent decreases in TRPV1 and TRPV3 mRNA levels, highly variable gene expression results between individual heterozygous $\triangle$ SVA HEK293 clones were observed (Figure 4B). For example, TRPV1 expression did not change in clone 2, whereas clone 1 and clone 3 both showed a decrease in TRPV1 expression. When we measured TRPV3 levels, clone 1 showed a large increase, clone 2 showed a small increase, and clone 3 showed a small decrease $(p>0.05)$. No statistical significance was determined for either TRPV1 or TRPV3 expression, however the overall trend observed in heterozygous $\triangle$ SVA clones was an increase in mRNA expression variability in both genes.

Analysis of RNA-seq data had shown co-expression of TRPV1 and TRPV3 in multiple human tissues (Figure 2C), therefore the ratio of TRPV1:TRPV3 in unedited and edited cell lines was examined (Figure 4C). A weak positive correlation between TRPV1 and TRPV3 expression in unedited HEK293 cells was identified and a strong positive correlation in cells transfected with ntgRNAs (Figure 4D). It should be noted that a small decrease in $T R P V 3$ expression in one unedited replicate was observed, however TRPV1 expression 
remain consistent across all unedited replicates. Given the small range in expression values between replicates, this small decrease in TRPV3 was enough to decrease the strength of the positive correlation in unedited replicates. However, this trend of positive correlation in unedited cells was lost in all edited clones. Homozygous $\triangle$ SVA clones showed a weak negative correlation between TRPV1 and TRPV 3 expression and no correlation was observed in heterozygous $\triangle$ SVA clones (Figure 4D). Across individual homozygous $\triangle$ SVA clones consistently increased levels of TRPV1 compared to TRPV3 were observed, which was not seen in unedited cells, indicating that co-expression of TRPV1 and TRPV3 was disrupted in edited cells completely lacking the SVA insertion ( $p$-value $>0.05$ ) (Figure 4C). Due to the variability of TRPV1 and TRPV 3 expression in heterozygous $\triangle$ SVA clones, no clear directional change in ratio was evident however the overall trend was an increase in variability ( $p$-value $>0.05$ ). It should be acknowledged that the statistical power in this study was limited due to the number of biological replicates $(n=3)$. These data further support that the regulatory mechanism contributing to the co-expression of TRPV1 and $T R P V 3$ observed in unedited cells, was not maintained in the absence of the SVA.

\section{Discussion}

\subsection{Human Specific SVA Insertion at the TRPV1/TRPV3 Locus Identified as a Candidate CRE}

In this study, we identified an SVA (subclass D) at the TRPV1/TRPV3 intergenic region, which we hypothesized was functional as a human specific CRE following comparative gene expression analysis showing species differences in TRPV1 and TRPV3 regulation between human and mouse. The differential expression of TPRV 3 between human and mouse has been previously reported in the literature, with a focus on roles in the nervous system (e.g., expression in DRG) $[19,24]$, however the data analysis conducted here suggested a broader physiological role for both TRPV1 and TRPV3 in many more human tissues. The results from our reporter gene assays conducted in HEK293 showed a repressive effect of the SVA when cloned into the pGL3-P system. These findings were consistent with data previously published by our group, showing repressive effects of other SVAs cloned into the same pGL3P system when tested in clonal cell lines SH-SY5Y and SKNAS [11,12]. Furthermore, our data is also consistent with that published by Trizzino et al., who cloned SVAs into the pGL4.23 system and conducted reporter assays in HepG2 cells [13,14]. Furthermore, a study of repression in exogenous SVA reporter constructs showed repressive binding of the human silencing hub (HUSH) complex to the central VNTR, which induced methylation at the SV40 promoter [25]. Overall, trends from various reporter models show a repressive effect of SVAs. However, this does not necessarily reflect the endogenous role of SVAs in situ, as they are simplistic and cannot account for additional factors like chromatin structure or adjacent regulatory sequences. Interestingly, the ECR adjacent to the SVA displayed all the hallmarks of a regulatory domain, therefore we expected to see greater changes in luciferase than those observed in this experiment. Nevertheless, when comparing results from both the ECR and SVA in HEK293, the SVA exerted a stronger transcriptional effect, leading us to further investigate its potential function as a CRE using CRISPR/Cas9.

\subsection{CRISPR-Cas9 Deletion of SVA in HEK293 Generated Homozygous and Heterozygous Clones}

Using a dual-target CRISPR/Cas9 approach, we successfully generated edited HEK293 clones with deletion of all SVA alleles (homozygous $\triangle \mathrm{SVA}$ ) and clones demonstrating the presence and absence of SVA alleles (heterozygous $\triangle$ SVA). Attempts were made to also delete the ECR using CRISPR to assess for endogenous effects that were not apparent in the reporter gene assays, however this was unsuccessful. A limitation of this method was the low yield of clones with the desired genetic modifications. We determined an editing efficiency of $10 \%$ for heterozygous $\Delta$ SVA clones and $<2 \%$ for homozygous $\Delta$ SVA. The previously published TAF1 SVA study reported a modification efficiency of $30 \%$, but this was performed in patient derived cell lines which possessed only a single pathogenic SVA insertion polymorphism at the TAF1 gene [16], in comparison to the SVA at TRPV1/TRPV3 
which is not an insertion-based polymorphic variant and thus required deletion of more than one copy. The lower modification efficiencies for homozygous $\triangle$ SVA clones, highlighted the difficult nature of deleting both copies of large DNA sequences. However, sequencing across edited regions in homozygous $\triangle$ SVA clones did reveal a high level of modification accuracy using this dual-target CRISPR/Cas9 system.

\subsection{TRPV3 Expression Was Significantly Decreased in Homozygous $\triangle S V A$ Clones}

Following the deletion of all SVA alleles in homozygous $\triangle$ SVA clones, a significant decrease in TRPV3 mRNA expression was seen compared to unedited cells, indicating that the presence of the SVA is contributing to the expression of TRPV3 in HEK293. Whilst a significant decrease in TRPV1 was also observed in homozygous $\triangle$ SVA clones compared to unedited cells, the level of decrease was similar to HEK293 transfected with ntgRNA controls, therefore the effects of the SVA deletion on TRPV1 mRNA expression could not be determined. Nevertheless, co-expression of TRPV1 and TRPV3 is found in many human tissues, which is not true of mouse tissues (e.g., DRG) which have been shown to express TRPV1 but not TRPV3 (Figure 2C). These functional data lend support to the hypothesis that SVAs serve as newly evolved CREs in primate genomes and contribute to gene regulation in primate species $[13,14,17,26]$. When we compared the ratio of TRPV1:TRPV3 expression between unedited and homozygous $\triangle$ SVA clones, a greater decrease in TRPV3 expression compared to TRPV1 was observed. It should be noted however that the greater effect observed on TRPV3 should be considered within this model only, and that differential regulation of TRPV1 and TRPV3 may be possible in a tissue-specific and stimulus-inducible fashion. In this model however, it is interesting that closer proximity of the SVA was much closer to the promoter of TRPV3 $(\sim 5 \mathrm{~kb})$ than to that of TRPV1 $(\sim 27 \mathrm{~kb})$. A recent study supporting the role of SVAs as proximal CREs showed that silencing of SVAs via induction of H3K9me3 marks, resulted in greater deregulation of genes with TSSs in close proximity $(0-5 \mathrm{~kb})$ to the SVA, than compared to genes with TSSs situated farther away $(>100 \mathrm{~kb})$ [26]. An alternative explanation is that the SVA is in even closer proximity (400 bp) to the $3^{\prime} \mathrm{UTR}$ of TRPV1. The $5^{\prime}$ end of the SVA contains a CT rich hexamer domain which contain MAZ-binding sites, which can affect polyadenylation signals and gene regulation-also in a tissue dependent manner [27]. Therefore, it would be interesting to quantify TRPV1 and TRPV3 protein levels to determine if there is any effect on mRNA stability and subsequent protein levels.

\subsection{TRPV1 and TRPV3 mRNA Expression Was Highly Variable in Heterozygous $\triangle S V A$ Clones}

Gene expression in heterozygous $\triangle$ SVA clones was highly variable, unlike homozygous $\triangle$ SVA clones that demonstrated consistent decreases in expression of TRPV1 and $T R P V 3$. No statistical significance was determined for either TRPV1 or TRPV3 in heterozygous $\triangle$ SVA clones, however the overall trend in heterozygous $\triangle$ SVA clones was an increase in mRNA expression variability of both genes. Furthermore, unlike homozygous $\Delta$ SVA clones, there was no correlation between gene expression values in heterozygous $\Delta$ SVA clones (Figure 4F). This indicated that loss of an SVA at the intergenic region between both genes may be disruptive to the regulatory mechanisms that drive co-expression in unedited cells. Deletion breakpoints were consistent in all homozygous $\triangle$ SVA clones but variable in heterozygous $\triangle$ SVA clones. It is hypothesized that this may have contributed to the observed differences. Consistent with this hypothesis, heterozygous $\triangle$ SVA clone 3 carried the same breakpoints in the edited region as all homozygous $\triangle$ SVA clones and showed expression of TRPV1 and TRPV3 that was consistent with the homozygous $\triangle$ SVA clones. However, heterozygous clones 1 and 2 showed increases specifically in TRPV3 expression. Interestingly, heterozygous clones 1 and 2 carried breakpoints in the edited region that resulted in a slightly larger deletion, which extended $10 \mathrm{bp}$ into a (CA)n repeat. This suggested that there was potentially unresolved regulatory potential in this additional sequence. Consideration must also be paid to the remaining SVA alleles in heterozygous $\triangle$ SVA clones. PCR genotyping indicated that an SVA allele was still present, however this 
did not guarantee that it was unedited. For example, DSBs could have been created and then repaired, failing to result in excision of the SVA, however indels or inversions could have occurred. We sequenced across breakpoints of the remaining SVA alleles following the CRISPR modification process. Annotated DNA sequences are given in Supplementary File 5. We found SNPs and a lack of alignment in clone 1 and 2 with the reference genome (Figure S3), indicating introduction and repair of DSBs resulting in the SVA-containing sequence being retained, however some additional modifications had also taken place. The effect of these modifications is unclear; nevertheless, whilst not statistically significant, a clear trend emerged in heterozygous $\triangle$ SVA clones showing deregulation in both TRPV1 and TRPV3 expression when compared to unedited cells and the ratio of TRPV1:TRPV3 was disrupted in heterozygous $\triangle$ SVA clones, consistent with homozygous $\triangle$ SVA clones.

\subsection{CRISPR Deletion of SVA at TRPV1/TRPV3 Locus Demonstrates in Situ Function as Newly} Evolved CRE

Overall, when taking all the data into account, deregulation of expression of TRPV1 and TRPV3 was observed in edited cells, regardless of homozygous or heterozygous $\triangle$ SVA deletions. The impact on gene expression following deletion of the SVA, supports its role as a CRE at the intergenic region between TRPV1 and TRPV3. The mechanism by which an SVA was previously identified as regulatory in TAF1 was intron retention which resulted in a decrease in TAF1 expression [16]. This specific disease mechanism is a different context to that being explored here and whilst relevant, should be considered independently $[15,16]$. The exact mechanism by which the SVA at TRPV1/TRPV3 functions remains to be determined however previous studies point to the recruitment of transcription factors $[9,28]$. Aberrant expression of TRPV1 is implicated in multiple pain associated conditions including diabetic neuropathy [29], irritable bowel syndrome [30], chronic pancreatitis [31], vulvodynia [32], and TRPV3 is elevated in inflammatory skinrelated conditions like psoriasis [33,34]. Thus, TRPV1 and TRPV3 remain key targets for the development of pharmaceuticals [35]. However, there have been difficulties translating advances identified in preclinical studies utilizing mouse models [36]. To our knowledge, to date, no regulatory domain contributing to the human specific expression of TRPV3 has yet been identified $[37,38]$. There are reports of TRPV1 and TRPV3 forming heteromeric channels in humans which are hypothesized to contribute to the fine tuning of sensory inputs, therefore the influence of the SVA and its role in TRPV1/TRPV3 regulation may have contributed to this molecular phenotype in human tissues and contributed to difficulties in developing drugs that are translatable based on mouse models [39,40]. It must be noted that the intergenic region contained many other transposable elements (e.g., Alus) that may also be predicted to contribute to gene regulation in human and non-human primates $[19,24]$, therefore the SVA would be a contributor in part to the full regulatory network of TRPV1 and TRPV3 observed in humans. However, it remained our focus to study the impact of a fairly recent SVA insertion-in terms of human genome evolution. In conclusion, the work presented here is the first reported example of a non-disease SVA being deleted using CRISPR and functional data supporting its role as a cis-regulatory domain that directly impacts mRNA expression of adjacent genes in vitro. To our knowledge, this is the first description of a human specific regulatory domain identified at the $T R P V 1$ and TRPV3 locus with the potential to contribute to the human specific expression of TRPV3 previously reported in the literature. These data give support to the role of SVAs as drivers of gene regulation and phenotypic evolution in primates, and shed light on the regulatory differences already identified between mice and humans at the TRPV1 and TRPV3 locus.

\section{Materials and Methods}

\subsection{Bioinformatic Analysis}

A list of SVA enriched regions was obtained from supplementary data published by Gianfrancesco et al. 2019 [4]. A list of all protein coding genes at the SVA enriched region at 
chr17p13.2-3, specifically at coordinates chr17:3000001-4000000 (hg19) were obtained from UCSC genome browser. The same coordinates were used to view the syntenic gene region in the mouse genome at coordinates chr11:72843250-74363624 (mm10). A list of protein coding genes was exported from UCSC from both human and mouse genomes at these coordinates. The list of protein coding genes was filtered for 1:1 orthologues. Expression values for 1:1 orthologues across multiple tissues in humans and mice was obtained from the RNA-seq dataset published by Ray et al. 2018 (given as transcripts per million; TPM) [21]. Data for comparable tissues across human and mouse were filtered out and used for cross species comparison. Heatmaps reflecting relative TPM values across species were generated using Morpheus software, available at https:/ / software.broadinstitute.org/morpheus / (accessed on 11 November 2020).

\subsection{Cell Culture}

Both luciferase reporter assays and CRISPR modifications were conducted in HEK293 cells (ATCC CRL-1573). Cells were cultured in Dulbecco's Modified Eagle Medium containing $4.5 \mathrm{~g} / \mathrm{L}$ D-glucose and L-glutamine (Gibco, Paisley, UK) supplemented with; 10\% FBS (Gibco), 1\% $100 \mathrm{mM}$ sodium pyruvate (Sigma, UK) and 1\% penicillin/streptomycin solution $(10,000$ units penicillin and $10 \mathrm{mg}$ streptomycin $/ \mathrm{mL}$ ) (Sigma). Cells were maintained in a humidified incubator at $37^{\circ} \mathrm{C}$ with $5 \% \mathrm{CO}_{2}$.

\subsection{Generating SVA and ECR Reporter Gene Constructs}

The SVA and ECR sequences were amplified from HEK293 gDNA using PCR. PCR reactions consisted of the following reagents; $1 \times$ Green GoTaq reaction buffer (Promega, Southampton, UK), $2.5 \mathrm{mM} \mathrm{MgCl}$ (Promega), $0.2 \mathrm{mM}$ dNTPs (Sigma), primers $0.1 \mathrm{mM}$ (Sigma), 0.5 units GoTaq Hot Start Polymerase (Promega), gDNA template (10 ng), made up to a final volume of $20 \mu \mathrm{L}$ with UltraPure ${ }^{\mathrm{TM}}$ DNase/RNase Free Distilled Water (Thermo Fisher Scientific, Gloucester, UK). Thermal cycles were performed using the SimpliAmp ${ }^{\mathrm{TM}}$ Thermal Cycler (Applied Biosystems, UK). Specific primers pairs and cycling conditions are listed in Table S1. PCR products were visualized using 1-2\% agarose gels stained with ethidium bromide solution (Sigma). Gels were visualized under UV light using the BioDocIt Imaging System (UVP). Amplicons and restriction digest products were purified using the Wizard ${ }^{\circledR}$ SV Gel and PCR Clean-Up System (Promega). Amplicons were initially ligated into the Dual promoter pCRII vector using the TA Cloning Kit (Invitrogen, Paisley, UK). The amplicon was then subcloned into the SacI and NheI sites of the reporter gene vector pGL3-Promoter (pGL3-P) (Promega), which encodes Firefly luciferase (FLuc). Ligations were completed using T4 DNA ligase (NEB). All cloning was propagated using Subcloning efficiency DH $5 \alpha$ competent cells (Invitrogen), grown in LB medium supplemented with $100 \mu \mathrm{g} / \mathrm{mL}$ ampicillin. Plasmid DNA used for transfection was purified using the Plasmid Maxi Kit (QIAGEN, Manchester, UK).

\subsection{Luciferase Reporter Gene Assays}

To evaluate the potential regulatory transcriptional activity of the SVA and ECR sequences, HEK293 cells were co-transfected with the relevant reporter gene plasmids and pRL-TK which encodes Renilla luciferase (RLuc) to enable normalization for transfection efficiency, using TurboFect Transfection Reagent (Thermo Fisher Scientific). pGL3-Basic (pGL3-B), which contains no promoter and should not express luciferase, was used as a negative control in transfection experiments. Transfections were repeated in four separate biological controls. Media was replaced $4 \mathrm{~h}$ post transfection and cells were assayed $48 \mathrm{~h}$ post transfection. FLuc and RLuc activity was measured in cell lysates using the Dual-Glo Luciferase Assay System (Promega). Relative light units (RLU) were detected using the GloMax 96 Microplate Luminometer. RLUs for each transfected culture were normalized against negative controls. The adjusted ratio of FLuc/RLuc was calculated for each condition and expressed as normalized Firefly luciferase activity (averaged across four repeats). 


\subsection{CRISPR/Cas9 Nuclease-Mediated Genome Editing}

sgRNA sequences flanking the $5^{\prime}$ and $3^{\prime}$ end of the SVA were identified using http: / / crispr.mit.edu/ (accessed on 17 May 2018) based on the S. pyogenes Cas9 5'-NGG-3' PAM recognition sequence. Target sequences are given in Supplementary File 3. Suitable oligonucleotides 20 bases in length were modified by removing the PAM sequence at the $3^{\prime}$ end of the sense oligonucleotide, followed by addition of CACC at the $5^{\prime}$ end of the sense oligonucleotide, and addition of AAAC at the $5^{\prime}$ end of the antisense oligonucleotide to generate BbsI overhangs. Complimentary oligonucleotides were annealed together to create a double stranded insert which was then ligated into the BbsI-linearized pSpCas9(BB)-2AGFP plasmid, as described by Ran et al. 2012. After ligation, bacterial transformation and isolation of plasmid DNA using the Wizard Plus SV Miniprep DNA Purification System (Promega), desired sgRNA inserts were confirmed using Sanger sequencing. To enable excision of the SVA, two recombinant plasmids containing the desired sgRNA inserts to target the $5^{\prime}$ and $3^{\prime}$ end of the SVA were co-transfected into HEK293 cells using TurboFect (Thermo Fisher Scientific). Untransfected unmodified HEK293 were used as a negative control. Cells transfected with non-target gRNA Cas9 constructs (ntgRNAs) were used as an additional control to assess for potential confounding effects of transfecting cells with Cas9 machinery on gene expression. $72 \mathrm{~h}$ post transfection, gDNA was purified using the GenElute Mammalian Genomic DNA Miniprep Kit (Sigma). PCR was used to verify the presence or absence of the SVA within the transfected cultures. Cultures showing evidence of an excised SVA were plated at a density of 1000 cells per $10 \mathrm{~cm}$ dish, to allow growth of single cells into colonies. When colonies were visible, they were selected and grown in duplicate until $70 \%$ confluent. Cell lysates from one duplicate culture were prepared for use as a direct template using DirectPCR Lysis Reagent (Viagen, California, CA, USA) and screened for modifications using PCR genotyping. Primer sequences and thermal cycles are given in Supplementary File 3. Successful excision of the SVA was determined using gel electrophoresis. Complete deletions were sequence verified using the forward PCR primer used in PCR genotyping. Sequencing was conducted externally by Source Bioscience (UK).

\section{6. $q P C R$}

Total RNA was extracted from HEK293 cell cultures using the Monarch Total RNA Miniprep Kit (NEB) and treated with DNaseI (Thermo Fisher Scientific). cDNA was synthesised using GoScript Reverse Transcriptase Kit (Promega). Quantitative PCR (qPCR) was performed on the Stratagene Mx3005P Real-Time PCR System (Agilent, Crawley, UK) using the GoTaq qPCR Master Mix (Promega). Reactions were set up in triplicate. ACTB was used as a reference gene. Target genes were TRPV1 and TRPV3. Relative quantification of target genes was calculated against the reference gene using the delta-delta $\mathrm{Ct}(2-\Delta \Delta \mathrm{Ct})$ method. Statistical analysis was performed using the 2-sample $t$-test in Minitab version 19.

Supplementary Materials: Supplementary materials can be found at https:/ / www.mdpi.com/14 22-0067/22/4/1911/s1. Supplementary file 1. Figure S1. UCSC screenshots showing overview of SVA insertions at chr17p13.2 with respect to the nearest protein coding genes. Supplementary file 2. DNA sequences of cloned fragments used in reporter gene assays. Supplementary file 3. Table S1. Oligonucleotide sequences and PCR thermal cycling conditions. Supplementary file 4. Figure S2. Validation of RT-PCR used to quantify gene expression. Supplementary file 5. Sequence reads across predicted double strand breakpoints in remaining SVA alleles following CRISPR modification. Supplementary file 6. Figure S3. Sequence alignment of double strand breakpoints in remaining SVA alleles against the reference human genome in UCSC browser.

Author Contributions: Conceptualization, E.P., V.J.B., and J.P.Q.; methodology, E.P., J.P.Q., V.J.B., O.G., and P.T.H.; writing-review and editing, E.P., B.F., P.T.H., V.J.B., and J.P.Q.; funding acquisition, J.P.Q., V.J.B., and B.F. All authors have read and agreed to the published version of the manuscript.

Funding: This research was funded by The Pain Relief Foundation (registered charity number: 1156227).

Institutional Review Board Statement: Not applicable. 
Informed Consent Statement: Not applicable.

Data Availability Statement: Not applicable.

Conflicts of Interest: The authors declare no conflict of interest. The funders had no role in the design of the study; in the collection, analyses, or interpretation of data; in the writing of the manuscript, or in the decision to publish the results.

$\begin{array}{ll}\text { Abbreviations } \\ \text { Cas9 } & \text { CRISPR associated protein 9 } \\ \text { CRE } & \text { cis-regulatory element } \\ \text { CRISPR } & \text { clustered regularly interspaced short palindromic repeats } \\ \text { DSB } & \text { double strand break } \\ \text { ECR } & \text { evolutionary conserved region } \\ \text { gDNA } & \text { genomic DNA } \\ \text { gRNA } & \text { guide RNA } \\ \text { HUSHiPSCs } & \text { human silencing hubinduced pluripotent stem cells } \\ \text { LINE-1 } & \text { long interspersed nuclear element 1 } \\ \text { LTR } & \text { long terminal repeat } \\ \text { MIR } & \text { mammalian interspersed repeat } \\ \text { NHEJ } & \text { non-homologous end joining } \\ \text { ntgRNA } & \text { non-target guide RNA } \\ \text { PAM } & \text { protospacer adjacent motif } \\ \text { PCR } & \text { polymerase chain reaction } \\ \text { qPCR } & \text { quantitative polymerase chain reaction } \\ \text { RLU } & \text { relative light unit } \\ \text { sgRNA } & \text { short guide RNA } \\ \text { SINE } & \text { short interspersed nuclear element } \\ \text { SVA } & \text { SINE-VNTR-Alu } \\ \text { TE } & \text { transposable element } \\ \text { TFBS } & \text { transcription factor binding site } \\ \text { TSS } & \text { transcriptional start site } \\ \text { UTR } & \text { untranslated region } \\ \text { UV } & \text { ultraviolet } \\ \text { VNTR } & \text { variable number tandem repeat } \\ \text { XDP } & \text { X-linked dystonia parkinsonism } \\ & \end{array}$

\section{References}

1. Wang, H.; Xing, J.; Grover, D.; Hedges, D.J.; Han, K.; Walker, J.A.; Batzer, M.A. SVA elements: A hominid-specific retroposon family. J. Mol. Biol. 2005, 354, 994-1007. [CrossRef]

2. Hancks, D.C.; Kazazian, H. SVA retrotransposons: Evolution and genetic instability. Semin. Cancer Biol. 2010, $20,234-245$. [CrossRef]

3. Tang, W.; Mun, S.; Joshi, A.; Han, K.; Liang, P. Mobile elements contribute to the uniqueness of human genome with 15,000 human-specific insertions and $14 \mathrm{Mbp}$ sequence increase. DNA Res. 2018, 25, 521-533. [CrossRef]

4. Schmidt, D.; Schwalie, P.; Wilson, M.; Ballester, B.; Gonçalves, Â.; Kutter, C.; Brown, G.; Marshall, A.; Flicek, P.; Odom, D. Waves of Retrotransposon Expansion Remodel Genome Organization and CTCF Binding in Multiple Mammalian Lineages. Cell 2012, $148,335-348$.

5. Tang, W.; Liang, P. Comparative Genomics Analysis Reveals High Levels of Differential Retrotransposition among Primates from the Hominidae and the Cercopithecidae Families. Genome Biol. Evol. 2019, 11, 3309-3325. [CrossRef]

6. Sundaram, V.; Cheng, Y.; Ma, Z.; Li, D.; Xing, X.; Edge, P.; Snyder, M.P.; Wang, T. Widespread contribution of transposable elements to the innovation of gene regulatory networks. Genome Res. 2014, 24, 1963-1976. [CrossRef] [PubMed]

7. Savage, A.L.; Schumann, G.G.; Breen, G.; Bubb, V.J.; Al-Chalabi, A.; Quinn, J.P. Retrotransposons in the development and progression of amyotrophic lateral sclerosis. J. Neurol. Neurosurg. Psychiatry 2019, 90, 284-293. [CrossRef] [PubMed]

8. Bourque, G.; Burns, K.H.; Gehring, M.; Gorbunova, V.; Seluanov, A.; Hammell, M.; Imbeault, M.; Izsvák, Z.; Levin, H.L.; Macfarlan, T.S.; et al. Ten things you should know about transposable elements. Genome Biol. 2018, 19, 1-12. [CrossRef] [PubMed]

9. Nikitin, D.; Penzar, D.; Garazha, A.; Sorokin, M.; Tkachev, V.; Borisov, N.; Poltorak, A.; Prassolov, V.; Buzdin, A.A. Profiling of Human Molecular Pathways Affected by Retrotransposons at the Level of Regulation by Transcription Factor Proteins. Front. Immunol. 2018, 9, 30. [CrossRef] 
10. Chuong, E.B.; Elde, N.C.; Feschotte, C. Regulatory evolution of innate immunity through co-option of endogenous retroviruses. Science 2016, 351, 1083-1087.

11. Savage, A.L.; Bubb, V.J.; Breen, G.; Quinn, J.P. Characterisation of the potential function of SVA retrotransposons to modulate gene expression patterns. BMC Evol. Biol. 2013, 13, 101.

12. Savage, A.L.; Wilm, T.P.; Khursheed, K.; Shatunov, A.; Morrison, K.E.; Shaw, P.J.; Shaw, C.E.; Smith, B.; Breen, G.; Al-Chalabi, A.; et al. An evaluation of a SVA retrotransposon in the FUS promoter as a transcriptional regulator and its association to ALS. PLoS ONE 2014, 9, e90833. [CrossRef] [PubMed]

13. Trizzino, M.; Park, Y.; Holsbach-Beltrame, M.; Aracena, K.; Mika, K.; Caliskan, M.; Perry, G.H.; Lynch, V.J.; Brown, C.D. Transposable elements are the primary source of novelty in primate gene regulation. Genome Res. 2017, 27, 1623-1633. [CrossRef] [PubMed]

14. Trizzino, M.; Kapusta, A.; Brown, C.D. Transposable elements generate regulatory novelty in a tissue-specific fashion. BMC Genom. 2018, 19, 468. [CrossRef]

15. Bragg, D.C.; Mangkalaphiban, K.; Vaine, C.A.; Kulkarni, N.J.; Shin, D.; Yadav, R.; Dhakal, J.; Ton, M.L.; Cheng, A.; Russo, C.T.; et al. Disease onset in X-linked dystonia-parkinsonism correlates with expansion of a hexameric repeat within an SVA retrotransposon in TAF1. Proc. Natl. Acad. Sci. USA 2017, 114, E11020-E11028. [CrossRef] [PubMed]

16. Rakovic, A.; Domingo, A.; Grutz, K.; Kulikovskaja, L.; Capetian, P.; Cowley, S.A.; Lenz, I.; Bruggemann, N.; Rosales, R.; Jamora, D.; et al. Genome editing in induced pluripotent stem cells rescues TAF1 levels in X-linked dystonia-parkinsonism. Mov. Disord. 2018, 33, 1108-1118. [CrossRef]

17. Gianfrancesco, O.; Geary, B.; Savage, A.L.; Billingsley, K.J.; Bubb, V.J.; Quinn, J.P. The Role of SINE-VNTR-Alu (SVA) Retrotransposons in Shaping the Human Genome. Int. J. Mol. Sci. 2019, 20, 5977.

18. Caterina, M.J.; Schumacher, M.A.; Tominaga, M.; Rosen, T.A.; Levine, J.D.; Julius, D. The capsaicin receptor: A heat-activated ion channel in the pain pathway. Nature 1997, 389, 816-824. [CrossRef]

19. Smith, G.D.; Gunthorpe, M.J.; Kelsell, R.E.; Hayes, P.D.; Reilly, P.; Facer, P.; Wright, J.E.; Jerman, J.C.; Walhin, J.P.; Ooi, L.; et al. TRPV3 is a temperature-sensitive vanilloid receptor-like protein. Nature 2002, 418, 186-190. [CrossRef]

20. Jjingo, D.; Conley, A.B.; Wang, J.; Mariño-Ramírez, L.; Lunyak, V.V.; Jordan, I.K. Mammalian-wide interspersed repeat (MIR)derived enhancers and the regulation of human gene expression. Mob. DNA 2014, 5, 14. [CrossRef]

21. Ray, P.; Torck, A.; Quigley, L.; Wangzhou, A.; Neiman, M.; Rao, C.; Lam, T.; Kim, J.Y.; Kim, T.H.; Zhang, M.Q.; et al. Comparative transcriptome profiling of the human and mouse dorsal root ganglia: An RNA-seq-based resource for pain and sensory neuroscience research. Pain 2018, 159, 1325-1345. [CrossRef]

22. Hollywood, J.A.; Lee, C.M.; Scallan, M.F.; Harrison, P.T. Analysis of gene repair tracts from Cas9/gRNA double-stranded breaks in the human CFTR gene. Sci. Rep. 2016, 6, 32230. [CrossRef]

23. Ran, F.A.; Hsu, P.D.; Wright, J.; Agarwala, V.; Scott, D.A.; Zhang, F. Genome engineering using the CRISPR-Cas9 system. Nat. Protoc. 2013, 8, 2281-2308. [CrossRef]

24. Xu, H.; Ramsey, I.S.; Kotecha, S.A.; Moran, M.M.; Chong, J.A.; Lawson, D.; Ge, P.; Lilly, J.; Silos-Santiago, I.; Xie, Y.; et al. TRPV3 is a calcium-permeable temperature-sensitive cation channel. Nature 2002, 418, 181-186. [CrossRef]

25. Robbez-Masson, L.; Tie, C.H.C.; Conde, L.; Tunbak, H.; Husovsky, C.; Tchasovnikarova, I.A.; Timms, R.T.; Herrero, J.; Lehner, P.J.; Rowe, H.M. The HUSH complex cooperates with TRIM28 to repress young retrotransposons and new genes. Genome Res. 2018, 28, 836-845. [CrossRef] [PubMed]

26. Pontis, J.; Planet, E.; Offner, S.; Turelli, P.; Duc, J.; Coudray, A.; Theunissen, T.W.; Jaenisch, R.; Trono, D. Hominoid-Specific Transposable Elements and KZFPs Facilitate Human Embryonic Genome Activation and Control Transcription in Naive Human ESCs. Cell Stem Cell 2019, 24, 724-735.e725. [CrossRef]

27. Himeda, C.L.; Ranish, J.A.; Hauschka, S.D. Quantitative proteomic identification of MAZ as a transcriptional regulator of muscle-specific genes in skeletal and cardiac myocytes. Mol. Cell. Biol. 2008, 28, 6521-6535. [CrossRef]

28. Pugacheva, E.M.; Teplyakov, E.; Wu, Q.; Li, J.; Chen, C.; Meng, C.; Liu, J.; Robinson, S.; Loukinov, D.; Boukaba, A.; et al. The cancer-associated CTCFL/BORIS protein targets multiple classes of genomic repeats, with a distinct binding and functional preference for humanoid-specific SVA transposable elements. Epigenetics Chromatin 2016, 9, 35. [CrossRef]

29. Facer, P.; Casula, M.A.; Smith, G.D.; Benham, C.D.; Chessell, I.P.; Bountra, C.; Sinisi, M.; Birch, R.; Anand, P. Differential expression of the capsaicin receptor TRPV1 and related novel receptors TRPV3, TRPV4 and TRPM8 in normal human tissues and changes in traumatic and diabetic neuropathy. BMC Neurol. 2007, 7, 11. [CrossRef] [PubMed]

30. Akbar, A.; Yiangou, Y.; Facer, P.; Walters, J.R.; Anand, P.; Ghosh, S. Increased capsaicin receptor TRPV1-expressing sensory fibres in irritable bowel syndrome and their correlation with abdominal pain. Gut 2008, 57, 923-929. [CrossRef] [PubMed]

31. Zhu, Y.; Colak, T.; Shenoy, M.; Liu, L.; Pai, R.; Li, C.; Mehta, K.; Pasricha, P.J. Nerve growth factor modulates TRPV1 expression and function and mediates pain in chronic pancreatitis. Gastroenterology 2011, 141, 370-377. [CrossRef]

32. Tympanidis, P.; Casula, M.A.; Yiangou, Y.; Terenghi, G.; Dowd, P.; Anand, P. Increased vanilloid receptor VR1 innervation in vulvodynia. Eur. J. Pain 2004, 8, 129-133. [CrossRef]

33. Nair, R.P.; Duffin, K.C.; Helms, C.; Ding, J.; Stuart, P.E.; Goldgar, D.; Gudjonsson, J.E.; Li, Y.; Tejasvi, T.; Feng, B.J.; et al. Genome-wide scan reveals association of psoriasis with IL-23 and NF-kappaB pathways. Nat. Genet. 2009, 41, 199-204. [CrossRef] 
34. Szollosi, A.G.; Vasas, N.; Angyal, A.; Kistamas, K.; Nanasi, P.P.; Mihaly, J.; Beke, G.; Herczeg-Lisztes, E.; Szegedi, A.; Kawada, N.; et al. Activation of TRPV3 Regulates Inflammatory Actions of Human Epidermal Keratinocytes. J. Invest. Dermatol. 2018, 138, 365-374. [CrossRef]

35. Damann, N.; Bahrenberg, G.; Stockhausen, H.; Habermann, C.J.; Lesch, B.; Frank-Foltyn, R.; Lee, J.; Ann, J.; Christoph, T. In vitro characterization of the thermoneutral transient receptor potential vanilloid-1 (TRPV1) inhibitor GRTE16523. Eur. J. Pharmacol. 2020, 871, 172934. [CrossRef]

36. Kym, P.R.; Kort, M.E.; Hutchins, C.W. Analgesic potential of TRPV1 antagonists. Biochem. Pharmacol. 2009, 78, 211-216. [CrossRef] [PubMed]

37. Fischer, M.J.M.; Ciotu, C.I.; Szallasi, A. The Mysteries of Capsaicin-Sensitive Afferents. Front. Physiol. 2020, 11, 554195. [CrossRef]

38. Gavva, N.R.; Treanor, J.J.; Garami, A.; Fang, L.; Surapaneni, S.; Akrami, A.; Alvarez, F.; Bak, A.; Darling, M.; Gore, A.; et al. Pharmacological blockade of the vanilloid receptor TRPV1 elicits marked hyperthermia in humans. Pain 2008, 136, 202-210. [CrossRef]

39. Cheng, W.; Yang, F.; Takanishi, C.L.; Zheng, J. Thermosensitive TRPV channel subunits coassemble into heteromeric channels with intermediate conductance and gating properties. J. Gen. Physiol. 2007, 129, 191-207. [CrossRef]

40. Cheng, W.; Yang, F.; Liu, S.; Colton, C.K.; Wang, C.; Cui, Y.; Cao, X.; Zhu, M.X.; Sun, C.; Wang, K.; et al. Heteromeric heat-sensitive transient receptor potential channels exhibit distinct temperature and chemical response. J. Biol. Chem. 2012, 287, 7279-7288. [CrossRef] [PubMed] 